\title{
Multiple Exciton Generation in InAs Nanocrystals
}

\author{
P. Kowalski AND P. MaChNiKOWsKI
}

Institute of Physics, Wrocław University of Technology

Wybrzeże Wyspiańskiego 27, 50-370 Wrocław, Poland

\begin{abstract}
We study a simple theoretical model of multiple exciton generation by a single high-energy photon absorbed in an InAs nanocrystal. We calculate the Coulomb matrix element for the electron-trion coupling in an InAs nanocrystal and show that due to the resulting coupling between single-pair and two-pair states the latter becomes weakly optically active.
\end{abstract}

PACS numbers: 78.67.Bf, 73.22.Dj

\section{Introduction}

Limitations of the efficiency of the existing solar cells motivate continuous search for new technological solutions that might lead to more efficient photovoltaic conversion. Among systems that are investigated for possible use in solar energy conversion devices are semiconductor quantum dots. A few ways of employing these nanometer-scale objects in the photoconversion process have been proposed $[1,2]$, one of which is multiple exciton generation in nanocrystals. Such an effect, consisting in generation of two or more electron-hole pairs by a single absorbed photon, has been observed in a few recent experiments performed on nanocrystals formed of various narrow-gap semiconductors [3-5]. It is a matter of discussion whether the excitons are created coherently during the absorption process or incoherently, due to carrier kinetics involving some kind of dissipative processes at a later time. In any case, the process results from the Coulomb coupling between electron and trion states. As a result, an intraband transition (relaxation) of the electron may be accompanied by the creation of another electron-hole pair, as shown in Fig. 1a. This Auger-type process is referred to as impact ionization. Let us note that the hole, which is initially created in the same confined state as the electron, does not take part in the impact ionization process.

In this paper we formulate and study a simple theoretical model of coherent multiple exciton generation by a single high-energy photon absorbed in an InAs nanocrystal. We calculate the interband Coulomb matrix element between an excited electron state and a ground state trion. We determine the degree of mixing 


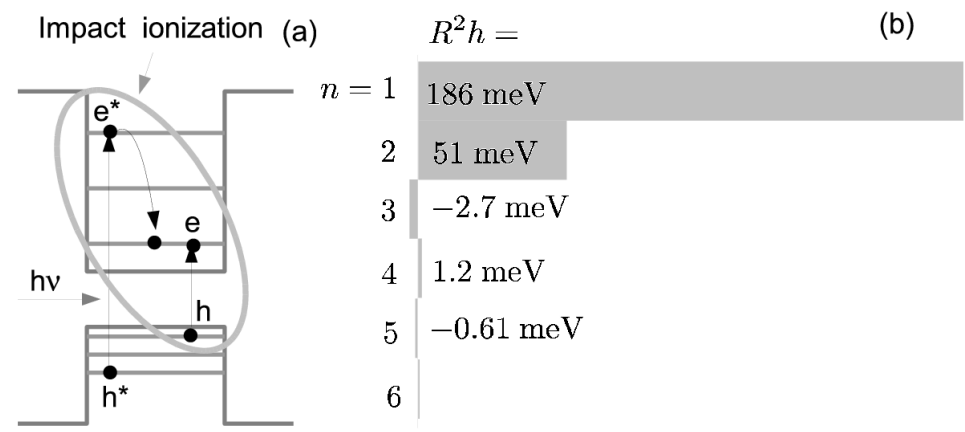

Fig. 1. (a) The diagram representing the process of multiple exciton generation via impact ionization in a nanocrystal. (b) Magnitudes of the matrix elements $h$ coupling an electron in the state $n, l=1, m=1$ with a trion state in which all carriers are in the ground state. The matrix element itself is proportional to $R^{-2}$.

between these states which results in a partial transfer of the oscillator strength for the optical transition from the single pair (exciton) state to a two-pair (biexciton) state.

\section{Model}

The InAs nanocrystal is modeled as a spherical potential well of radius $R$ with infinite potential walls. In the single band envelope function approximation, the wave functions are

$$
\Psi_{\sigma, \nu}(\boldsymbol{r}, s)=u_{\sigma}(\boldsymbol{r}, s) \psi_{\nu}(\boldsymbol{r}) .
$$

Here $u_{\sigma}(\boldsymbol{r}, s)$ is the Bloch function for the band $\sigma$, position $\boldsymbol{r}$ and spin $s$. The index $\nu$ denotes the whole set of the usual quantum numbers of a spherically symmetric problem, $\nu=(n l m)$. The envelope is given by

$$
\psi_{n l m}(\boldsymbol{r})=\frac{1}{R^{3 / 2}} N_{n l} Y_{l}^{m}(\theta, \phi) j_{l}\left(x_{l n} r / R\right),
$$

where

$$
N_{n l}= \begin{cases}{\left[-\frac{1}{2} j_{l-1}\left(x_{l n}\right) j_{l+1}\left(x_{l n}\right)\right]^{-1 / 2},} & l>0, \\ x_{l n} / \sqrt{2}, & l=0,\end{cases}
$$

$Y_{l}^{m}$ is a spherical harmonic, $j_{l}$ is a spherical Bessel function, and $x_{n l}$ is the $n$-th zero of $j_{l}$. In our model, we neglect band mixing and the Coulomb interaction between electrons and holes.

The corresponding energy levels for electrons and holes are

$$
E_{n l}^{(\mathrm{e}, \mathrm{h})}=\frac{\hbar^{2}}{2 m_{\mathrm{e}, \mathrm{h}} R^{2}} x_{l n}^{2} .
$$

We take the heavy hole effective mass $m_{\mathrm{h}}=0.35 m_{0}$ and use an implicit formula for the energy-dependent electron mass in the decoupled bands approximation [6]: 


$$
m_{\mathrm{e}}=m_{0}\left(\alpha+\frac{E_{\mathrm{P}}}{E_{\mathrm{g}}+E_{n l}}\right)^{-1}
$$

where $E_{\mathrm{P}}=22.2 \mathrm{eV}, E_{\mathrm{g}}=0.418 \mathrm{eV}$ is the (bulk) band gap, and the parameter $\alpha=0.77$ accounts for the coupling to higher bands. Using the implicit formula for the electron and a constant effective mass for the hole is motivated by the small effective mass of the former which leads to larger kinetic energies, compared to a hole.

The electron-trion coupling responsible for the impact ionization process and for the transfer of oscillator strength from a single-pair state to a two-pair state results from the Coulomb interaction. The relevant Hamiltonian is

$$
\begin{aligned}
H= & \frac{e^{2}}{4 \pi \epsilon_{0} \epsilon_{\mathrm{s}}} \sum_{\nu_{1} \nu_{2} \nu_{3} \nu_{4}} \sum_{s s^{\prime}} \sum_{\lambda \sigma_{1} \sigma_{2} \sigma} \int \mathrm{d}^{3} \boldsymbol{r} \int \mathrm{d}^{3} \boldsymbol{r}^{\prime} \Psi_{\mathrm{c} \sigma_{1} \nu_{1}}^{*}\left(\boldsymbol{r}^{\prime}, s^{\prime}\right) \Psi_{\mathrm{c} \sigma_{2} \nu_{2}}^{*}(\boldsymbol{r}, s) \\
& \times \frac{1}{\left|\boldsymbol{r}^{\prime}-\boldsymbol{r}\right|} \Psi_{\mathrm{v} \lambda \nu_{3}}(\boldsymbol{r}, s) \Psi_{\mathrm{c} \sigma \nu_{4}}\left(\boldsymbol{r}^{\prime}, s^{\prime}\right) a_{\mathrm{c} \sigma_{1} \nu_{1}}^{\dagger} a_{\mathrm{c} \sigma_{2} \nu_{2}}^{\dagger} a_{\mathrm{v} \lambda \nu_{3}} a_{\mathrm{c} \sigma \nu_{4}}+\text { h.c. },
\end{aligned}
$$

where we have added an additional label explicitly specifying the band ("v" valence, "c" - conduction). We assume that the initial excitation transfers an electron from a heavy hole valence band state with the spin $3 / 2$ to a conduction band state with spin $1 / 2$. In order to avoid Pauli blocking for the impact ionization transition to the ground state, we assume that the spins of the electron-hole pair created in this process are opposite to those of the original pair. Thus, we set $\lambda=-3 / 2$ and $\sigma_{1}=1 / 2$, hence $s=-1 / 2$ and $\sigma_{2}=\sigma=s^{\prime}=1 / 2$.

By using the identity

$$
\frac{1}{\left|\boldsymbol{r}^{\prime}-\boldsymbol{r}\right|}=\frac{1}{(2 \pi)^{3}} \int \mathrm{d}^{3} \boldsymbol{q} \frac{4 \pi}{q^{2}} \mathrm{e}^{\mathrm{i} \boldsymbol{q} \cdot\left(\boldsymbol{r}^{\prime}-\boldsymbol{r}\right)}
$$

and performing the standard separation between the integration over a unit cell and the summation over the nanoscopic scale of the nanocrystal, one finds the relevant matrix element of $H$ in the form

$$
\begin{aligned}
& h_{\nu_{1} \nu_{2} \nu_{3} \nu_{4}}=\delta_{m_{1}+m_{2}, m_{3}+m_{4}+1} 2^{3 / 2} \pi \\
& \quad \times \frac{e^{2}}{4 \pi \epsilon_{0} \epsilon_{s}} \frac{d_{0}}{R^{2}} \int \mathrm{d} \vartheta \sin ^{2} \vartheta \int \mathrm{d} u u \mathcal{F}_{\nu_{1} \nu_{4}}(u, \vartheta) \mathcal{F}_{\nu_{3} \nu_{2}}^{*}(u, \vartheta) .
\end{aligned}
$$

Here $\boldsymbol{d}_{0}$ is proportional to the interband dipole moment,

$$
\boldsymbol{d}_{0}=\int_{\text {e.c. }} \mathrm{d}^{3} \boldsymbol{r} u_{\mathrm{c},-1 / 2}^{*}(\boldsymbol{r}) \boldsymbol{r} u_{\mathrm{v},-3 / 2}(\boldsymbol{r})
$$

(for InAs, we estimate $\boldsymbol{d}_{0}=1.9 \mathrm{~nm}$ ) and the form-factors are

$$
\begin{aligned}
& \mathcal{F}_{\nu, \nu^{\prime}}(u, \vartheta)=K_{l m} K_{l^{\prime} m^{\prime}} N_{n l} N_{n^{\prime} l^{\prime}} \int \mathrm{d} y y^{2} \int \mathrm{d} \theta \sin \theta P_{l}^{m}(\cos \theta) P_{l^{\prime}}^{m^{\prime}}(\cos \theta) \\
& \times j_{l}\left(x_{l n} y\right) j_{l^{\prime}}\left(x_{l^{\prime} n^{\prime}} y\right) \mathrm{e}^{\mathrm{i} u y \cos \theta \cos \vartheta} J_{m-m^{\prime}}(u y \cos \theta \cos \vartheta),
\end{aligned}
$$


where

$$
K_{l m}=\sqrt{\frac{(2 l+1)(l-m) !}{4 \pi(l+m) !}},
$$

$J_{m}$ is a Bessel function and $P_{l}^{m}$ are associated Legendre polynomials.

We calculate the matrix element coupling a high-energy electron state to a trion state in which all the particles are in the ground state (corresponding to the process shown in Fig. 1a). Equivalently, this may be interpreted as coupling a photocreated electron-hole pair with both carriers in a state $(n l m)$ to a two-pair state with a hole in the state $(\mathrm{nlm})$ and the three other carriers in the ground state (000). This matrix element is non-zero only for $l=1, m=1$. The result for a few lowest values of $n$ is shown in Fig. 1b. The matrix element is large for low states, where it can reach tens of $\mathrm{meV}$ for small nanocrystals. However, it decreases very quickly for higher states.

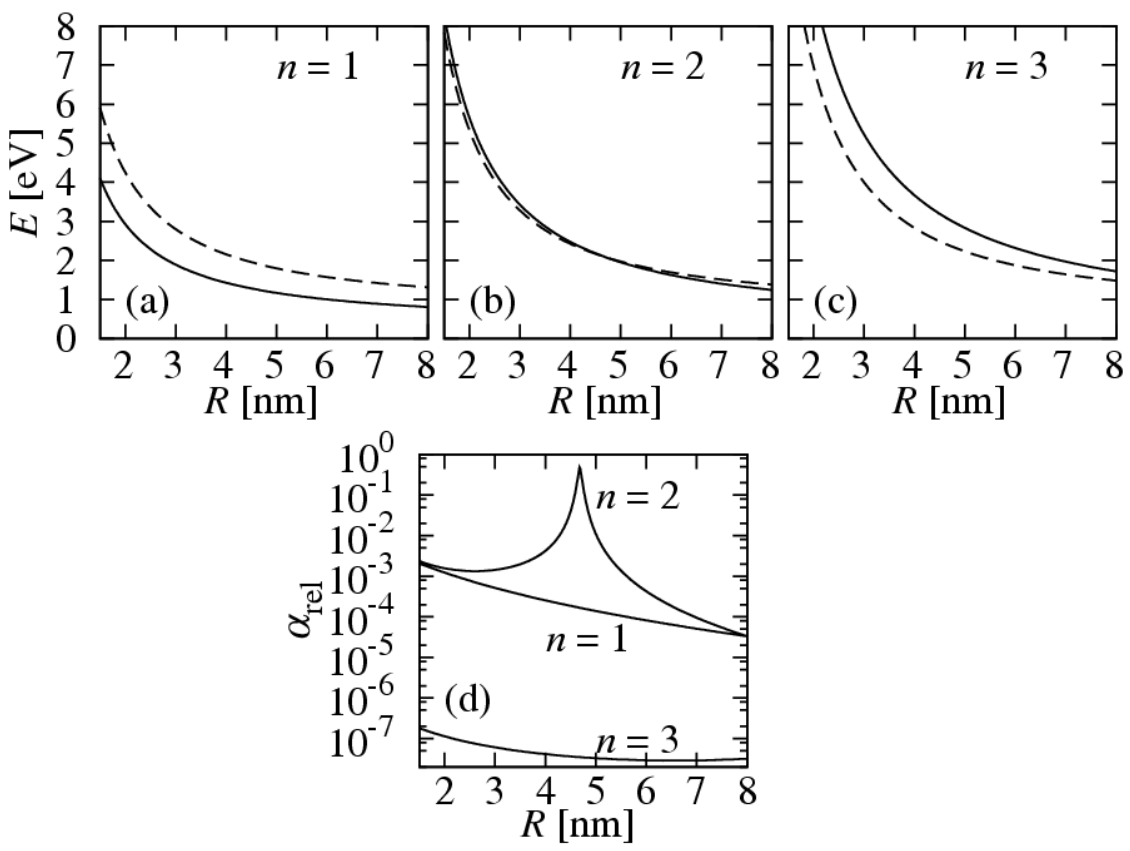

Fig. 2. (a)-(c) Energies of single-pair and two-pair states coupled by the interband Coulomb matrix element for the radial quantum number of the single pair state as shown. (d) The relative intensity of the optical transition to the predominantly twopair states.

In Fig. 2a-c we show the energies of the pairs of coupled states for $n=1,2,3$. Here the solid lines represent the states of predominantly single-exciton character, while the dashed lines correspond to the predominantly two-pair states. As can be seen in Fig. 1b, the states with $n=1$ are strongly coupled but differ considerably 
in energy, as shown in Fig. 2a. States with $n=2$ are much closer and cross at $R \approx 5 \mathrm{~nm}$ (Fig. 2b), hence a stronger transfer of the oscillator strength can be expected here. States with $n>2$ (Fig. 2c) are distant in energy and weakly coupled, so one can expect no effect.

In Fig. 2d we plot the relative intensity for the optical transition, calculated as the square of the interband dipole moment attained by the two-pair states as a result of the coupling to the optically active single-exciton state. In the case of the ground state $(n=1)$, the transfer is weak, but it could still lead to efficient excitation of the two-pair state for sufficiently intense light if there are no competing single-exciton transitions in the same spectral range. Strong transfer is observed for the $n=2$ state in the area of crossing between the single-pair and two-pair energies.

\section{Discussion and conclusions}

We have formulated and studied a theoretical model of multiple exciton generation by a single high-energy photon absorbed in an InAs nanocrystal. The importance of the multiple exciton generation lies in the fact that it can lead to an increased current per absorbed photon and therefore to an improved efficiency in nanocrystal-based solar cells of a new generation. We have calculated the Coulomb matrix element for the electron-trion coupling responsible for the multiple exciton generation. We have shown that this matrix element is proportional to the interband dipole moment between the conduction and valence bands of a bulk crystal and inversely proportional to the square of the crystal radius.

Even though the coupling can be as strong as tens of $\mathrm{meV}$ in small nanocrystals between states with the same radial quantum number, the separation between the energies of the one-pair and two-pair states is usually much larger so that, typically, the two-pair state becomes only very weakly optically active. Still, efficient multiple exciton generation should be possible for sufficiently intense incident light if there are no competing single-exciton transitions in the same spectral range. Such a weak, off-resonant coupling depends rather weakly on the nanocrystal size.

Stronger coupling is possible in the area of the crossing between the single-pair and two-pair energies. However, such a resonance condition is not a generic feature and appears only for a specific nanocrystal size. One should note also that at the exact resonance both states are equally mixed and correspond to 1.5 pairs on the average.

Our results show that a process consisting in the coherent generation of two excitons in low energy states of a nanocrystal by a single high-energy photon seems to be possible. They suggest also that the efficiency of this process can be controlled by the engineering of electronic properties (band gap and intraband transition energies) of nanocrystals. 


\section{References}

[1] V. Aroutiounian, S. Petrosyan, A. Khachatryan, K. Tourian, J. Appl. Phys. 89, 2268 (2001).

[2] A.J. Nozik, Physica E 14, 115 (2002).

[3] R.D. Schaller, V.I. Klimov, Phys. Rev. Lett. 92, 186601 (2004).

[4] R.D. Schaller, J.M. Pietryga, V.I. Klimov, Nano Lett. 7, 3469 (2007).

[5] R.J. Ellingson, M.C. Beard, J.C. Johnson, P. Yu, O.I. Micic, A.J. Nozik, A. Shabaev, A.L. Efros, Nano Lett. 5, 865 (2005).

[6] A.L. Efros, M. Rosen, Phys. Rev. B 58, 7120 (1998). 